International Journal of Automotive and Mechanical Engineering (IJAME)

ISSN: 2229-8649 (Print); ISSN: 2180-1606 (Online);

Volume 13, Issue 3 pp. 3710 - 3727, December 2016

CUniversiti Malaysia Pahang Publishing

DOI: https://doi.org/10.15282/ijame.13.3.2016.14.0304

\title{
Heat transfer performance of non-circular coiled tubes - Research summary, challenges and directions
}

\author{
J.C. Kurnia ${ }^{1^{*}}$ and A.P. Sasmito ${ }^{2}$ \\ ${ }^{1}$ Department of Mechanical Engineering, Universiti Teknologi PETRONAS, \\ 32610 Seri Iskandar, Malaysia \\ *Email: jundika.kurnia@utp.edu.my \\ Phone: +605 368 7157; Fax: +605 3656461 \\ ${ }^{2}$ Department of Mining and Materials Engineering, \\ McGill University, 3450 University Street, Frank Dawson Adams Bldg, \\ Montreal Quebec H3A 2A7 Canada
}

\begin{abstract}
Coiled tubes have been widely used in major industries due to their high heat transfer, ease of manufacturing and compactness. Numerous studies have been conducted experimentally and numerically to evaluate the heat transfer performance of coiled tubes. A majority of these studies were focused on circular tubes, while only limited studies were concerned on non-circular tubes. Recently, a series of computational studies have been conducted in our research group to investigate the flow characteristic and heat transfer performance of non-circular coiled tubes. Several key parameters affecting flow behavior and heat transfer performance have been evaluated and examined. The heat transfer performance is numerically evaluated and compared by utilizing the concept of Figure of Merit (FoM). The paper is intended to provide an essential summary of these numerical studies with the aim of providing a general overview of the advantage and drawbacks of non-circular coiled tubes in thermal engineering applications. Challenges and directions of the research will also be discussed. In general, it is revealed that heat transfer improvement in the coiled tube is followed by increasing pumping power. Moreover, with a more complex coiled geometry proposed, the processes of manufacturing the tube and creating the computational domain will become major issues in non-circular coiled tube studies. In addition, more studies on the application of coiled tubes used for heat exchanger and chemical reactor are expected to increase in the future, especially in the micro or nanoscale.
\end{abstract}

Keywords: Coiled tube, heat transfer performance; numerical investigation research summary.

\section{INTRODUCTION}

Improving and intensifying heat transfer has been a major focus of engineering researchers worldwide. For this objective, significant efforts have been devoted with the ultimate goal of achieving the maximum transfer rate while keeping energy utilization at the minimum. Three methods are commonly adopted in enhancing heat transfer, i.e. passive method that does not require external power, active method which utilizes external power and a combination of both. Among the various passive methods, one promising method is the application of coiled tubes. For decades, coiled tubes have 
been a major focus in heat transfer engineering due to its high heat transfer rate and compactness [1-3]. Their unique characteristics have attracted considerable attention from researchers worldwide. A high heat transfer rate in a coiled tube is primarily attributed to the presence of a secondary flow induced by the coil curvature which enhances mixing and convection occurring in the region near the outer wall. Pioneer studies conducted by Dean revealed that a secondary flow initiated by the centrifugal force occurs in a Toroidal (in-plane) tube when the Dean Number exceeds a critical value $[4,5]$. Subsequent studies revealed that the secondary flow may be observed as one pair or two pairs depending on the Dean Number $[6,7]$. The effect of the secondary flow on the laminar heat transfer was studied by Dravid, Smith [8] who found that the heat transfer coefficient in helical tubes oscillates until a fully-developed flow is reached. This phenomenon is in line with findings by Akiyama and Cheng [9].

Aside from elaborating the basic mechanism of the secondary flow in coiled tubes and its heat transfer performance effect, numerous studies have focused on the effect of various key parameters affecting the flow and heat transfer in coiled tubes such as temperature dependent fluid thermal properties [10, 11], Reynolds number [10-12], curvature [11-13], and non-Newtonian fluid [14]. In addition, the application of coiled tubes in heat exchangers has been evaluated and reported. Rennie and Raghavan [15] [16] had computationally and experimentally studied the application of a curved tubein-tube design as a heat exchanger. They reported that the heat transfer performance increases as the Dean number increase. In addition, it was found that a counter flow provides higher heat transfer rates. Zachár [17] introduced a helical corrugation on the outer side of the helical heat exchanger to optimize the heat transfer performance. It was found that corrugation improves heat transfer up to 80-100\% while imposing a 10-600\% higher pressure drop. The majority of these studies were focused on circular crosssection. Among the few studies concerning non-circular coiled tubes, Egner and Burmeister [18] had evaluated the effect of the cross-section aspect ratio and Reynolds number of a rectangular curved duct and found that the maximum secondary velocity depends on the Reynolds number and aspect ratio. In addition, it was revealed that a combination of Reynolds number and curvature ratio determines the critical Dean number at which the secondary flow changes its pattern. Ko [19] numerically evaluated the heat transfer enhancement in a square coiled tube by introducing longitudinal ribs. The results indicated that flow characteristic and heat transfer are strongly influenced by the placement of longitudinal rib where a single rib arrangement shows superior performance compared to other configurations. Driven by the fact that studies on heat transfer performance of a non-circular coiled tube is scarce, our group have conducted and reported a series of computational studies [20-31] investigating the heat transfer performance of non-circular coiled tubes. Various coil geometries and cross-sections are evaluated to assess the heat transfer performance. Several key parameters affecting heat transfer performance have been evaluated [32]. The addition of nano particles and micro-encapsulated phase change material on the heat transfer fluid has also been investigated. In order to have a comprehensive and holistic overview on the heat transfer performance of non-circular tubes, it is therefore of interest to summarize the progress of research carried out and identify future research's direction and outlook.

This paper presents an overview and summary of numerical studies on the flow characteristic and heat transfer performance of non-circular coiled tubes that have been conducted in our group with the aim of providing guidelines on the applications of noncircular coiled tubes. To stimulate a better understanding of the studies, a brief overview on the computational fluid dynamic (CFD) model and constitutive relations used to 
evaluate heat transfer are presented. A brief description of the numerical implementation on the developed model will be presented. Challenges and future directions of research on non-circular coiled tubes will also be described and discussed to guide other researchers working on this topic.

\section{METHODS AND MATERIALS}

\section{Mathematical Modelling}

A series of studies have been conducted to investigate the flow characteristic and heat transfer performance in square helical tubes by utilizing a three dimensional CFD model for an incompressible laminar Newtonian fluid. In the following, a brief overview of the computational model used for the numerical studies is presented and discussed. More details on the computational model for each study are available in our previous publications [20-31].

\section{Governing Equations}

To initiate the studies, a three dimensional CFD model for an incompressible laminar flow inside straight and coiled tubes have been developed and validated against empirical correlations proposed by Edwards, Denny [33] and Baehr and Stephan [34] for straight tubes, and Manlapaz and Churchill [35] for coiled tubes as shown in Figure 1. The model comprises of conservation equations for mass, momentum, and energy, i.e.

$$
\begin{gathered}
\nabla \rho \cdot \mathbf{u}=0 \\
\nabla \cdot(\rho \mathbf{u} \otimes \mathbf{u})=-\nabla P+\nabla \cdot\left[\mu\left(\nabla u+(\nabla u)^{T}\right)\right] \\
\rho c_{p} \mathbf{u} \cdot \nabla T=k \nabla^{2} T
\end{gathered}
$$

where $\rho$ is fluid density, $\mathbf{u}$ is velocity, $P$ is pressure, $\mu$ is dynamic viscosity, $c_{p}$ is specific heat, $k$ representing thermal conductivity and variable $T$ is temperature[36, 37].

\section{Constitutive Relations}

The working fluids considered are air and water. Since the thermo physical properties of air and water vary with temperature, constant properties may not be sufficient to capture the real characteristics of the working fluid. Hence, the working fluid's properties are prescribed as a polynomial function of temperature. These polynomial functions were developed based on the air and water properties taken from Kays, Crawford [38]. As a matter of accuracy, an $\mathrm{R}^{2}$ value of more than 0.99 was obtained for all equations. The air properties are represented as the following [20]:

$$
\begin{gathered}
\rho_{a}=1.076 \times 10^{-5} T^{2}-1.039 \times 10^{-3} T+3.326 \\
\mu_{a}=5.21 \times 10^{-15} T^{3}-4.077 \times 10^{-11} T^{2}+7.039 \times 10^{-8} T+9.19 \times 10^{-7} \\
k_{a}=4.084 \times 10^{-10} T^{3}-4.519 \times 10^{-7} T+2.35 \times 10^{-4} T-0.0147 \\
c_{p, a}=-4.67 \times 10^{-6} T^{3}+4.837 \times 10^{-3} T^{2}-1.599 T+1175
\end{gathered}
$$

Meanwhile for water, the properties are given by [20] 


$$
\begin{gathered}
\rho_{w}=-3.570 \times 10^{-3} T^{2}+1.88 T+753.2 \\
\mu_{w}=2.591 \times 10^{-5} \times 10^{\frac{238.3}{T-143.2}} \\
k_{w}=-8.354 \times 10^{-6} T^{2}+6.53 \times 10^{-3} T-0.5981
\end{gathered}
$$

The specific heat for water, $c_{p, w}$ is constant at $4200 \mathrm{~J} / \mathrm{kg} \cdot \mathrm{K}$.
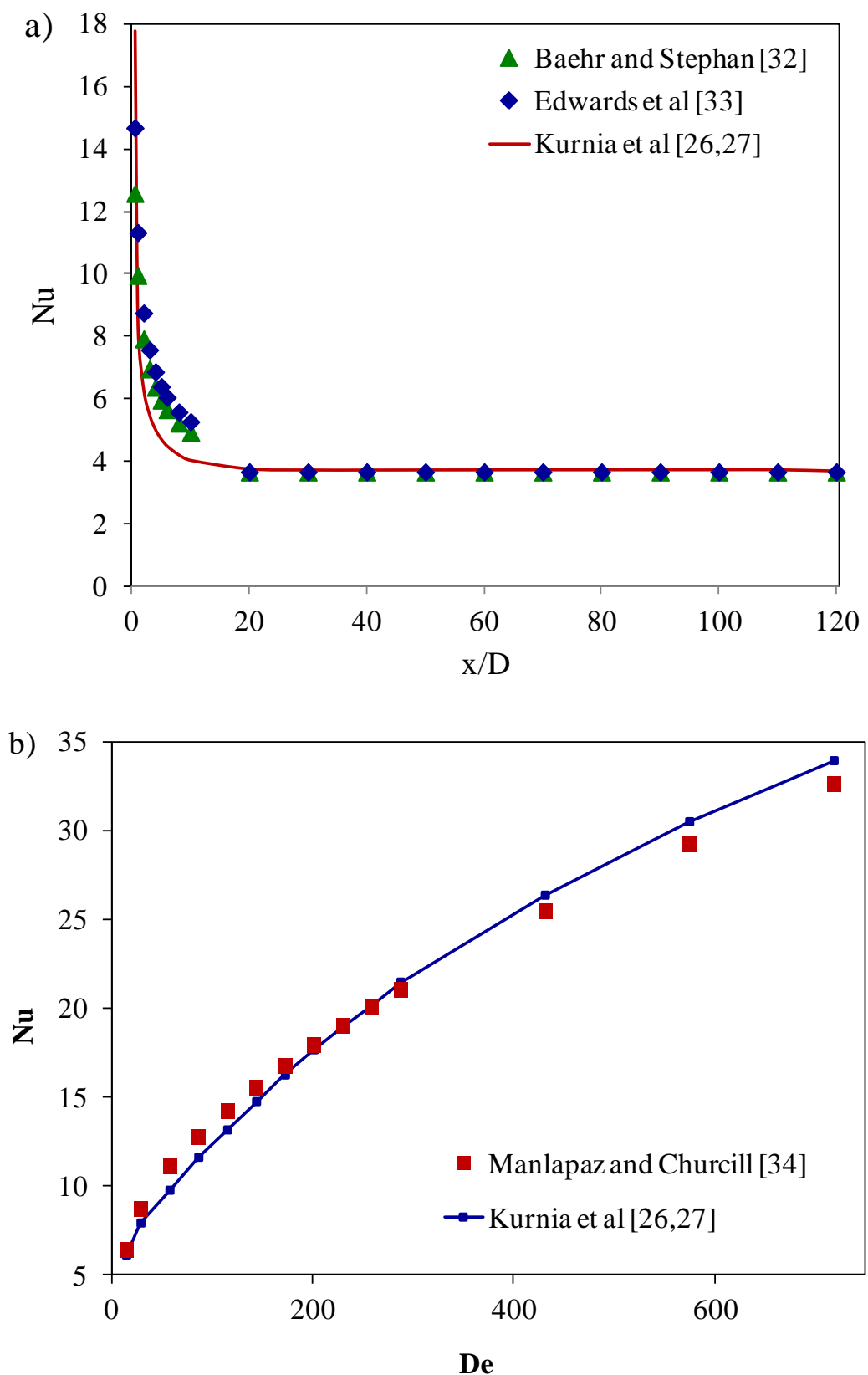

Figure 1. Comparison between model prediction developed in our previous study [26, $27]$ and correlations proposed by previous researchers $[33,35]$ for a) straight and b) coiled tubes. 
The heat transfer performance of the studied geometries were evaluated in terms of the mixed mean temperature along the tubes, $\mathrm{T}_{\text {mean }}$, total heat transfer, $\dot{Q}$, and FoM. The mixed mean temperature is given by [20]

$$
T_{\text {mean }}=\frac{1}{V A_{c}} \int_{A_{c}} \mathrm{u} T d A_{c}
$$

where $A_{c}$ is the cross section area of the tubes and $V$ is the mean velocity given by [20]

$$
V=\frac{1}{A_{c}} \int_{A_{c}} \mathrm{u} d A_{c}
$$

The total heat transfer rate and FoM are defined as [20, 26, 27]

$$
\begin{gathered}
\dot{Q}_{\text {total }}=\dot{m} c_{p}\left(T_{\text {mean }, L}-T_{\text {mean }, 0}\right) \\
F o M=\frac{\dot{Q}_{\text {total }}}{P_{\text {pump }}}
\end{gathered}
$$

where $\dot{m}$ is mass flow rate and $T_{\text {mean, } L}$ and $T_{\text {mean }, 0}$ are the mixed mean temperature at length $L$ and at the channel inlet, respectively. Pumping power, $P_{p u m p}$, is given by [[2527]

$$
P_{\text {pump }}=\frac{1}{\eta_{\text {pump }}} \dot{V} \Delta p
$$

with $\eta_{\text {pump }}$ being pump efficiency (assumed to be $70 \%$ ) and $\dot{V}$ being the volumetric flow rate of the working fluid. Two commonly encountered boundary conditions (constant wall temperature and constant wall heat flux) were investigated.

\section{Boundary Conditions}

The boundary conditions imposed on the computational model were varied according to the studied cases. However, the commonly used boundary conditions are listed as follows:

- Inlet: at the inlet, inlet velocity (sometime mass flow rate) which corresponds to studied Reynolds number and constant inlet temperature (and species if applicable) is prescribed

- Outlet: at the outlet, pressure and stream wise gradient of temperature (and species if applicable) are set to zero. The outlet velocity is unknown a priori and it needs to be calculated by iteration from the neighbouring computational cells.

- Walls: Non-slip conditions for velocity together with either constant wall temperature or constant wall heat flux are specified at the wall.

\section{Numerical Methodology}

The computational domains were created in AutoCAD software and then labelled and meshed in Gambit. The mathematical model together with constitutive relations and appropriate boundary conditions are then solved by using finite volume solver ANSYS Fluent. A mesh independence test was conducted to ensure mesh independent results [20-31]. On average, mesh amounts ranging from $2 \times 10^{5}$ to $5 \times 10^{5}$ are required to model 
the computational domain [20-31]. The equations were solved using Semi-Implicit Pressure Linked Equation algorithm [39], second-order upwind discretization and Algebraic Multi-grid method. The convergence criteria of all relative residuals are set as $10^{-6}$.

\section{Heat Transfer Performance Of Coiled Tubes}

Here, we will briefly summarize some important findings obtained from a series of studies on flow characteristic and heat transfer performance in a square helical tube by utilizing a three dimensional CFD model for an incompressible laminar Newtonian fluid which were conducted in our research group. More detailed discussion can be found in our previous publications [20-31].

\section{Coil Geometry}

The first study addresses the heat transfer performance of various configurations of coiled non-circular tubes, namely in-plane spiral, helical spiral, conical spiral and straight tubes as shown in Figure 2 [20]. Two heat transfer fluids (air and water) were evaluated to investigate the effect of Prandtl number. Both constant wall heat flux and constant wall temperature were investigated. The investigation was carried out for a Reynolds number range of 100-1000.

a)
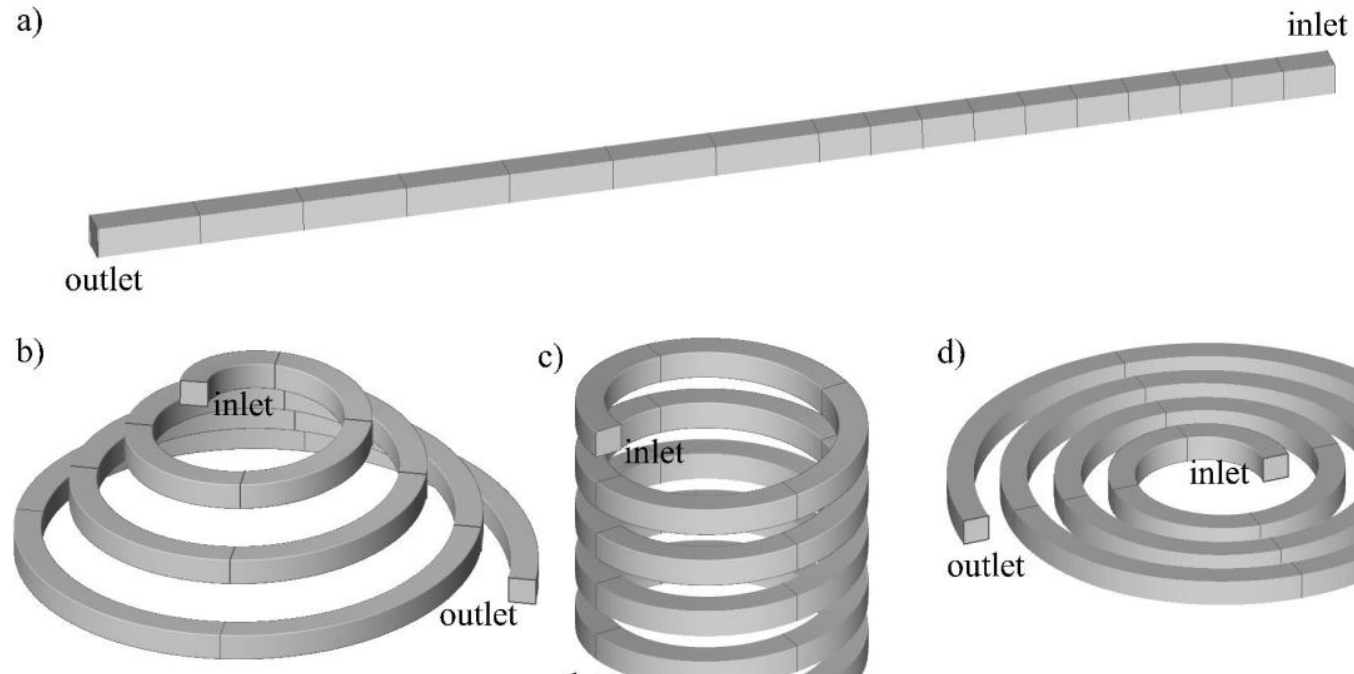

c)

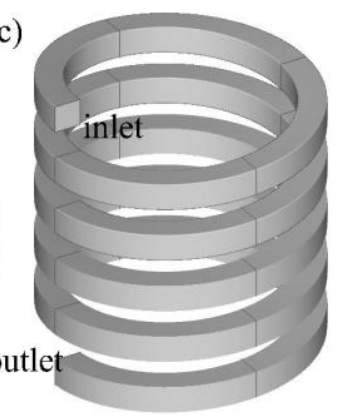

d)

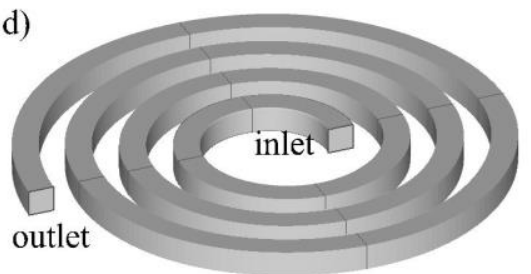

Figure 2. Schematic representations of a) straight, b) conical spiral, c) helical spiral, and d) in-plane spiral tubes [20].

It was found that the presence of a secondary flow significantly affects the heat transfer performance in coiled tubes as represented by temperature distribution in Figure 3. Higher temperature was observed in all coiled tubes. On closer inspection, higher temperature gradient is observed near the outer wall of the coiled tubes, indicating a higher transfer rate. This is mainly contributed by the secondary flow which is induced by centrifugal forces due to coil curvature. Overall, coiled tubes offer higher heat transfer at the cost of a significantly higher pressure drop (and thus pumping power). Among the studied geometries, the helical tube yields the highest heat transfer for a 
constant wall heat flux. Meanwhile, for constant wall temperature, both conical and inplane spiral offer superior performance.

Investigation on the effect of inlet Reynolds number (which directly corresponds to inlet velocity) revealed that higher Reynolds number results in higher heat transfer rates. This is expected since higher velocity drives more working fluids to absorb the heat from the tube wall. Comparing air and water for the same Re, it is clearly observed that water has superior heat transfer performance compared to air. As stated previously, Figure of Merit (FoM) is introduced to fairly compare the studied geometries. The result revealed that despite having the lowest heat transfer rate, the straight tube has the highest FoM owing to its significantly lower pressure drop. On the other hand, the helical tube has the lowest FoM in spite of its high heat transfer due to the high pressure drop.

a)

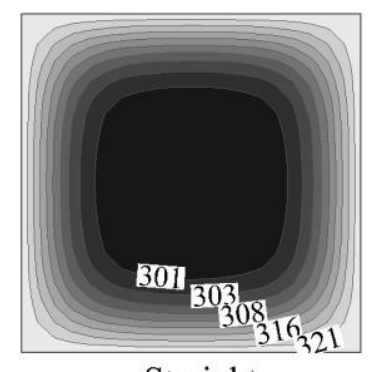

d)

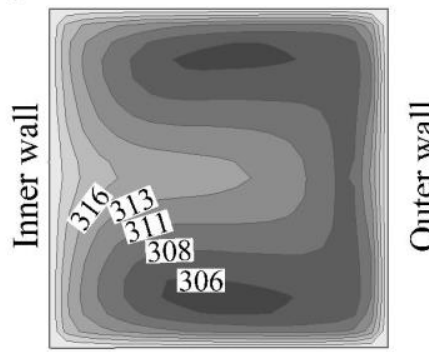

Helical spiral b)

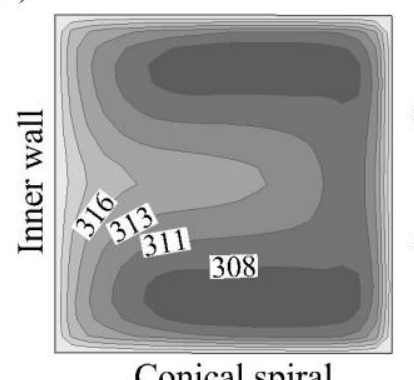

Conical spiral c)

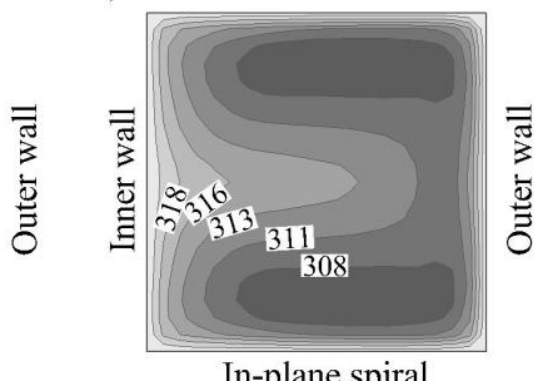

In-plane spiral

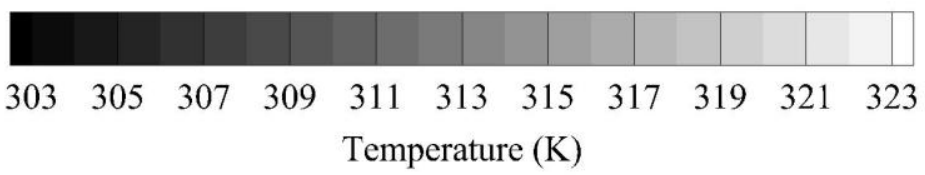

Figure 3. Temperature distribution of water flow at $\mathrm{L}=20 \mathrm{~cm}$ and $\mathrm{Re}=500$ [20].

From this study, it was found that for the coiled tube, after a certain length from the inlet, no more heat transfer occurs as the fluid temperature is equal to the wall temperature. It is therefore of interest to evaluate the heat transfer of a coiled tube with the specific length at which the maximum temperature is reached. By reducing the length of the coiled tube, a lower pressure drop (and thus pumping power) is expected; hence the Figure of Merit for this coiled tube can be increased. In addition, it will also offer savings in terms of material cost to produce the tube.

\section{Cross-section Geometry}

A further point of interest is the effect of various cross-sections geometries (rectangular, square, triangular, trapezoidal, circular and half circular) on the heat transfer performance of in-plane spiral tubes [21,22]. Similar to the previous study, water and air were considered as heat transfer fluids in this study. In addition, both the constant wall heat flux and constant wall temperature were evaluated. Several inlet velocities were selected to evaluate the effect of Reynolds number (100, 500 and 1000). The heat 
transfer performance of coiled tubes was compared with those of straight tubes with the same cross-section. The studied geometries are presented in Figure 4.
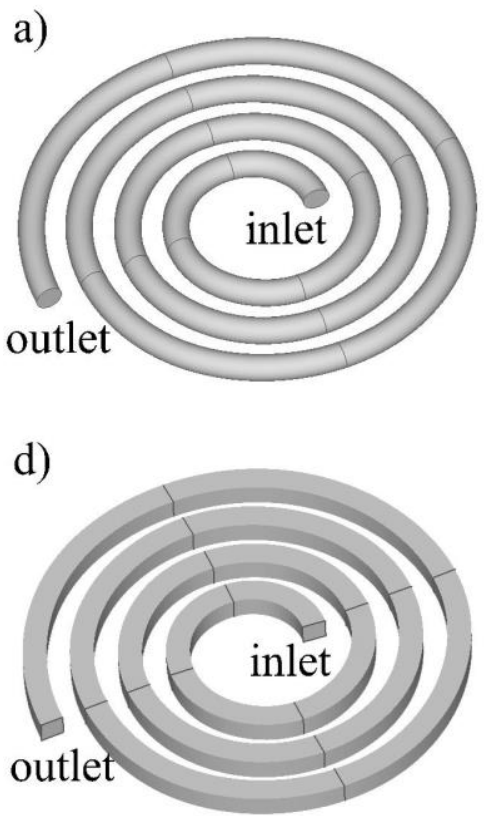

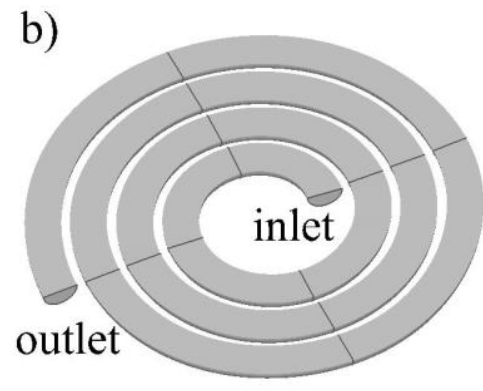

f)

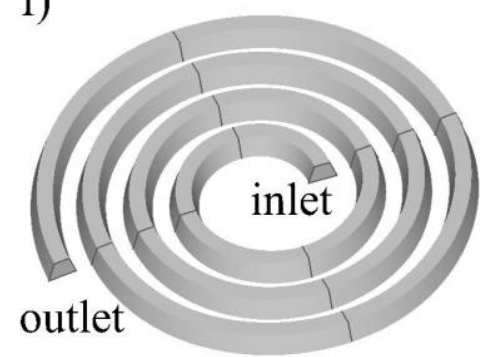

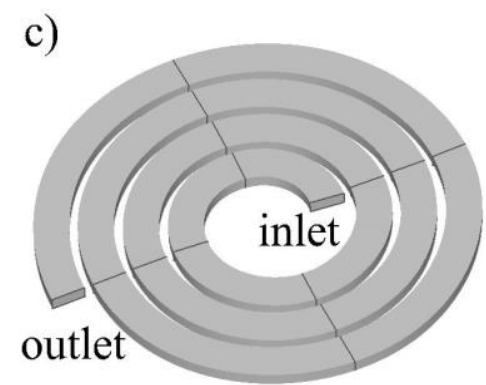

g)

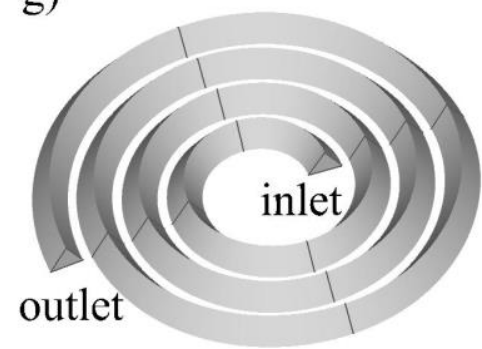

Figure 4. Schematic representations of in-plane spiral tubes with a) circular, b) halfcircular, c) rectangular, d) square, f) trapezoidal, and g) triangular cross-sections [21, 22].

a)

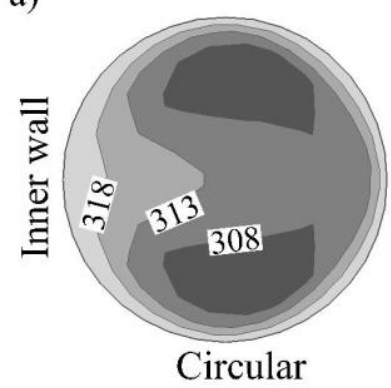

d)

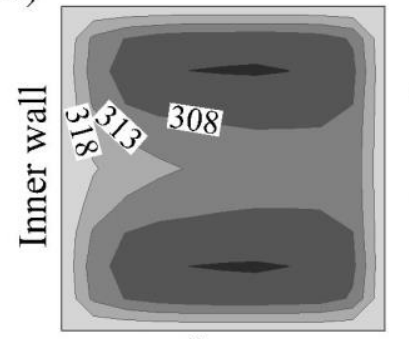

Square
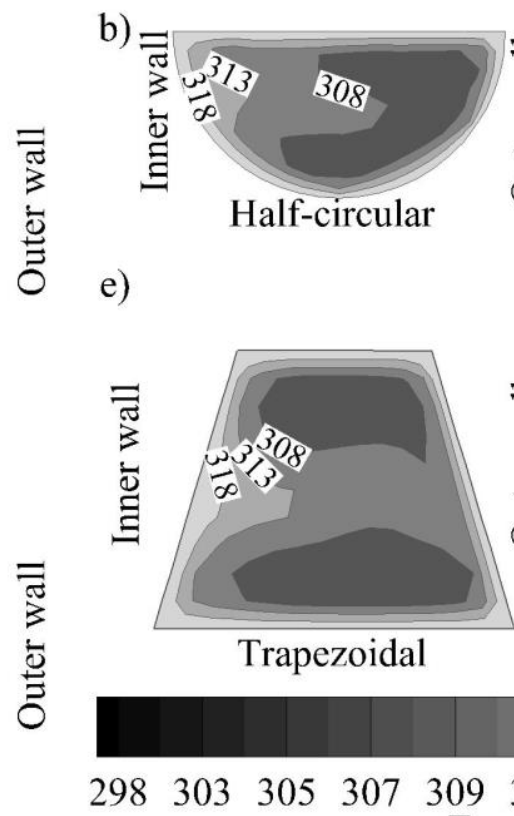

e)

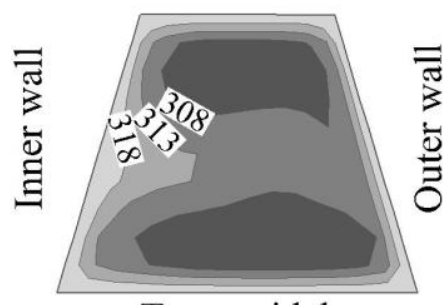

Trapezoidal

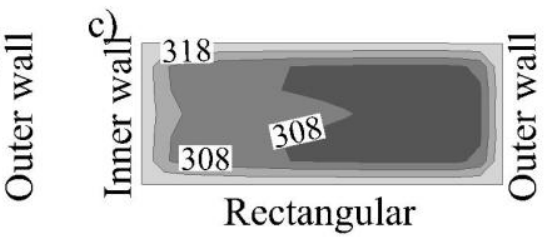

f)

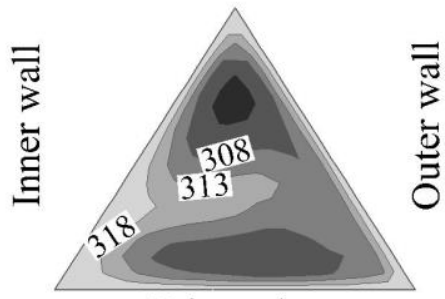

Triangular

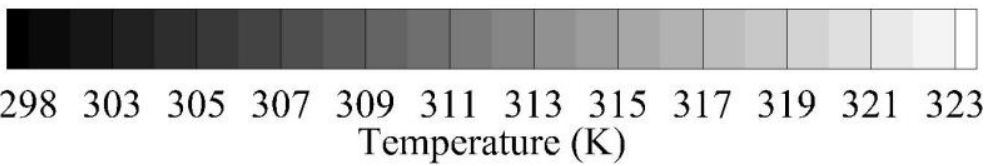

Figure 5. Temperature distribution of water flow at $\mathrm{L}=20 \mathrm{~cm}$ and $\mathrm{Re}=500$ inside inplane spiral tubes with various cross-sections [21, 22].

As presented in Figure 5, the cross section profile directly affects the flow behavior and heat transfer in a coiled tube. The results indicated that for both constant wall temperature and constant wall heat flux, in-plane spiral with rectangular and 
triangular cross-sections offers superior heat transfer rate. This is observed for both working fluids (air and water). With regards to pumping power requirements, it was found that an in-plane spiral with rectangular and triangular cross-sections requires higher pumping power compared to other cross-sections. Note that in this study, the inlet velocity and cross-section area are held constant. In terms of FoM, in line with the previous study, the in plane spiral tubes have lower FoM compared to straight tubes with the same cross-section due to the significantly higher pressure drop. Among the coiled tubes, the in-plane spiral with a circular cross-section offers the highest FoM due to its smaller pressure drop despite its inferior heat transfer rate compared to the other cross-sections.

While it is numerically possible to have various cross-section profiles for coiled tubes, manufacturing it for real applications may be impractical and expensive. Hence, for the time being, circular coiled tubes will remain the most commonly used coiled tube. In the future when manufacturing technology is more advanced, coiled tubes with other cross section geometries can be manufactured.

\section{Coil Curvature}

This study addresses the flow characteristic and heat transfer performance of a laminar flow $(\operatorname{Re}=500)$ inside helical tubes with various curvature diameters. Both constant wall temperature and constant wall heat flux were studied. Two working fluids (water and air) were evaluated. Three curvature radiuses $-3 \mathrm{~cm}, 4 \mathrm{~cm}$ and $5 \mathrm{~cm}$ - were evaluated and compared with straight tubes of identical cross-sections. The schematics of the studied geometries are presented in Figure 6.

a)

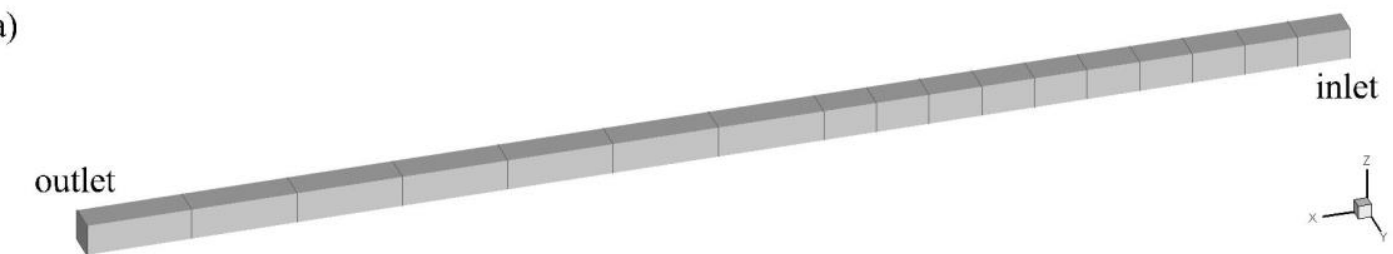

b)

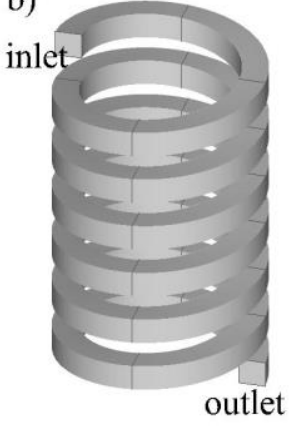

c)

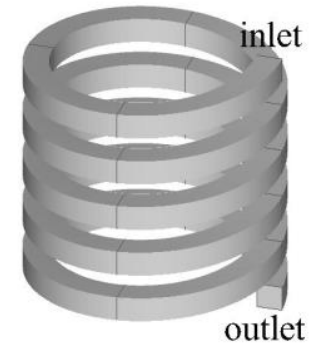

d)

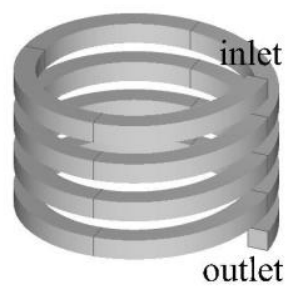

Figure 6. Schematic representations of a) straight tube and helical tubes with helical radii of: b) $3 \mathrm{~cm}$; c) $4 \mathrm{~cm}$; and d) $5 \mathrm{~cm}$.

In line with previous studies for circular cross-sections [11-13], a smaller curvature radius was found to offer a higher heat transfer rate. This is represented by a higher and more uniform temperature distribution inside a helical tube with smaller curvature radius as presented in Figure 7. It should be noted, however, that smaller curvature radius also imposes a higher pressure drop and in turn, higher pumping power 
requirements. In terms of FoM, a smaller curvature radius yields a lower FoM due to the higher pressure drop imposed.

a)

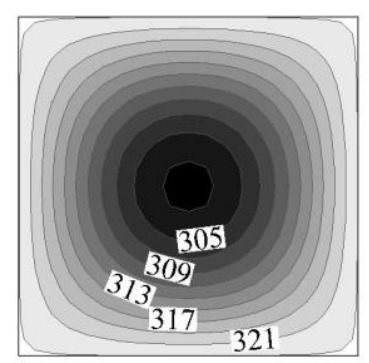

Straight

d)

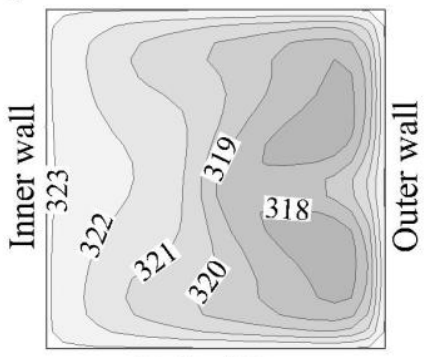

Helical $5 \mathrm{~cm}$ b)

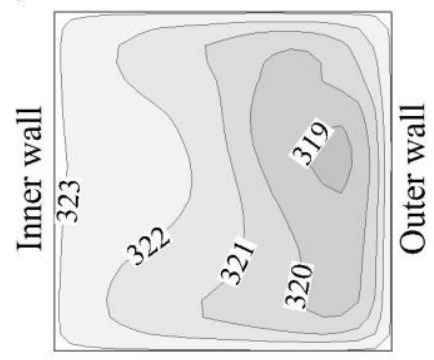

Helical $3 \mathrm{~cm}$ c)

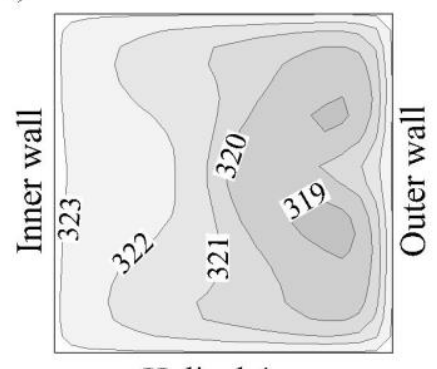

Helical $4 \mathrm{~cm}$

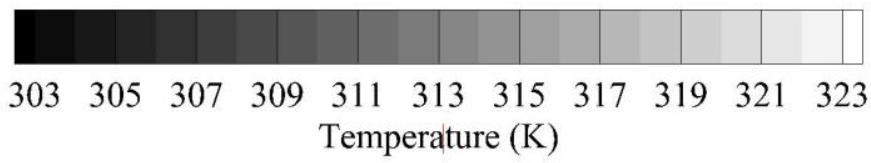

Figure 7. Temperature distribution in a straight tube and helical tubes with various helical radii at $\mathrm{L}=25 \mathrm{Re}=500$.

\section{Nanoparticle suspension}

This study is an extension of the first study where the heat transfer performance of various configurations of coiled non-circular tubes was evaluated at Reynolds number 1000. In this study, however, nanoparticle [23] and micro-encapsulated phase change material (MEPCM) [24] were added to the heat transfer fluid (water) in order to enhance the heat transfer rate. This study is motivated by the fact that there are numerous real-life applications where heat transfer enhancement by addition of nanoparticle to the base heat transfer fluid (such as water or glycol) is necessary, especially for cooling applications [40-42]. Referred to as nanofluid, this suspension offers a higher heat transfer rate compared to the traditional heat transfer fluid [23]. Several studies investigating the heat transfer performance of water-based $\mathrm{TiO}_{2}, \mathrm{SiO}_{2}$, $\mathrm{CuO}$ and $\mathrm{Al}_{2} \mathrm{O}_{3}$ nanofluids have been conducted and reported [43-47]. Their results indicate that higher heat transfer rates can be obtained by the addition of small amounts of nanoparticles. In our study [23], two nanoparticles were evaluated, i.e. $\mathrm{Al}_{2} \mathrm{O}_{3}$ and $\mathrm{CuO}$. While for the MEPCM suspension, the phase change material evaluated is paraffin wax (n-octadecane) [24].

The effect of nanoparticle and MEPCM concentration on the heat transfer performance of the coiled tubes was examined. It was found that a small addition of nanoparticles of up to $1 \%$ volumetric concentration is beneficial for heat transfer enhancement. However, when more nanoparticles are added to the heat transfer fluid, the heat transfer rate will decrease. In addition, adding large amounts of nanoparticles may lead to agglomeration and sedimentation which can further deteriorate the heat transfer inside the tubes. Comparing both nanoparticles, no significant difference was observed in terms of heat transfer rate. Both nanoparticle suspensions at $1 \%$ volumetric concentration enhanced the heat transfer by roughly $15 \%$ compared to that of base fluid 
(water). Hence, selection between the two nanoparticles will be focused on their stability and cost. It should be noted, however, that the addition of nanoparticles is only beneficial at low concentrations. Higher concentrations of nanoparticles will not only impose a higher pumping power, but also lead to agglomeration which will deteriorate the heat transfer performance. As such, careful consideration and examination are required to obtain the optimum concentration of nanoparticles that should be added to the base heat transfer fluid in order to enhance its heat transfer performance.

For the MEPCM suspension, it was revealed that increasing the MEPCM concentration does increase the heat transfer rate at the cost of a higher pumping power. The improvement which is due to the addition of MEPCM, however, is not significant as compared to the increase of pressure drop due to it. Among the studied coiled tubes, the in-plane spiral tubes yields the highest heat transfer rate and FoM, thus highlighting the potential of this tube in combination with MEPCM for heat exchanger applications. More studies are needed to evaluate the various parameters that may significantly affect heat transfer performance such as particle size, percentage of PCM and shells, and other related parameters.

\section{Non-Newtonian fluid}

This is another extension of the first study where the heat transfer performance of a carboxymethil cellulose (CMC) solution of power law non-Newtonian fluid flowing inside various configurations of coiled non-circular tubes at Reynolds number 1000 were evaluated [25]. Several CMC concentrations (100 ppm, 200 ppm, 500 ppm, 1000 ppm and $2000 \mathrm{ppm}$ ) were evaluated to investigate the effect of power law index and consistency index on heat transfer performance. In this study, constant wall temperature boundary conditions are prescribed.

The numerical results indicate that for the same inlet Reynolds number, the heat transfer rate of the CMC solution is up to two times of that of water. However, it also imposes as high as 4 times pressure drop as compared to water. Another finding is that the heat transfer rate and pressure drop are not linear to the $\mathrm{CMC}$ concentration: both increases up to 2 times when 100-200 ppm CMC is added. When more CMC is added to the solution (at $500 \mathrm{ppm}$ ), heat transfer and pressure drop slightly decrease, and increase again when the CMC is more than $1000 \mathrm{ppm}$. Overall, CMC has a smaller FoM compared to water. Among the CMC concentrations, 500 ppm has the highest FoM due to the smaller pressure drop imposed.

This study was limited to only one type of non-Newtonian fluid, i.e. power-law fluid. More studies investigating the heat transfer behavior of other non-Newtonian fluids are required to provide a comprehensive view on the heat transfer performance of these fluids in coiled tubes. Moreover, future studies can also investigate the optimum geometric and operating conditions.

\section{Large Temperature Difference}

In heat transfer applications, the heat transfer fluid inside a coiled tube will be subjected to large temperature difference. Hence, it is of interest to study the flow behavior and heat transfer performance of heat transfer fluids subjected to these large temperature difference conditions inside the coiled tubes. Several coil geometries (in-plane, conical, helical) and cross-sections (circular, ellipse, square) were examined [26, 27]. In addition to the first law thermodynamic analysis, an exergy analysis was conducted to evaluate the performance of the studied geometries based on the second law of thermodynamics. Similar to the previous studies, the concept of FoM is utilized to analyze the results. The 
effects of Reynolds number and wall temperature on heat transfer were examined as well. The results indicate that the coiled tube offers a higher transfer rate compared to its straight tube counterpart despite imposing a higher pressure drop. Interestingly, the coiled tube was found to generate lower entropy, indicating its high efficiency as compared to the straight tube. Among the coiled tubes, the in-plane tube yields the highest FoM. Higher wall temperature and Reynolds number were found to be beneficial for heat transfer performance. Meanwhile, for the studied cross-section, the square cross-section generates the highest entropy, followed by ellipse and circular. Moreover, it also imposes the highest pressure drop among the studied geometries. Nevertheless, the square cross-section offers the highest transfer rate. On another note, it is revealed that entropy generation is dominated by heat transfer, while entropy generation due to viscous dissipation contributes marginally to the total entropy generation. For the effect of cooling and heating, it was found that cooling offers a higher FoM, indicating better heat transfer performance when compared to heating, although it generates slightly higher entropy as well.

\section{Application in cooling channels of electronic components and polymer electrolyte fuel cells}

Previous studies have revealed that the coiled tube design offers higher heat transfer rates compared to the straight tube. It is therefore of interest to evaluate the performance of the coiled design in cooling channel applications for electronic components and polymer electrolyte fuel cells (PEFC) [28, 29]. The performance of coiled designs in dissipating heat from electronic components and PEFCs was evaluated and compared to those of the conventional parallel, serpentine and wavy designs as well as the newly proposed oblique fin design. The studied channel designs are presented in Figure 8.

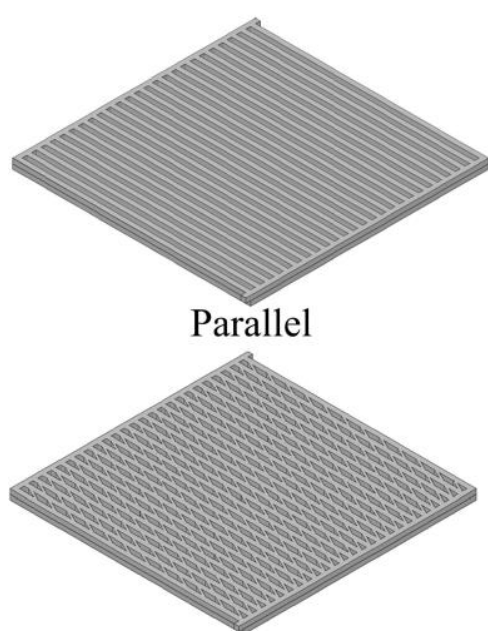

Parallel oblique

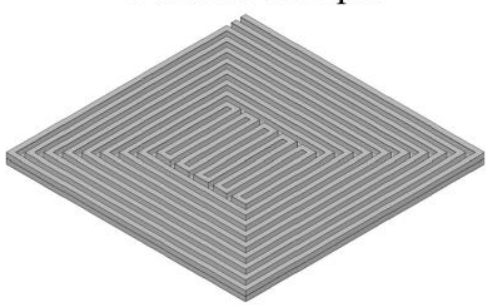

Coil serpentine
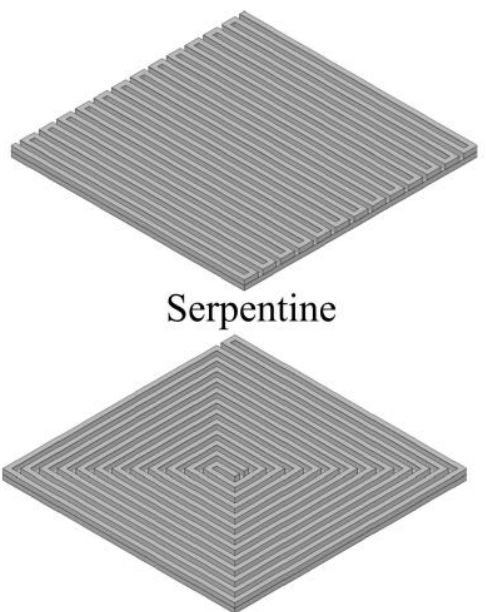

Coil inner inlet

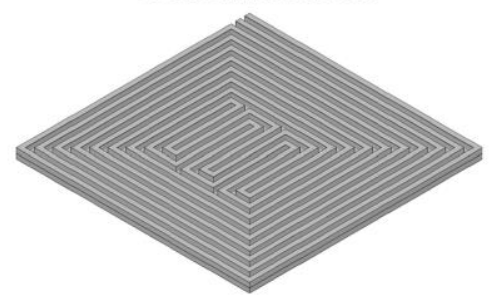

Coil double serpentine

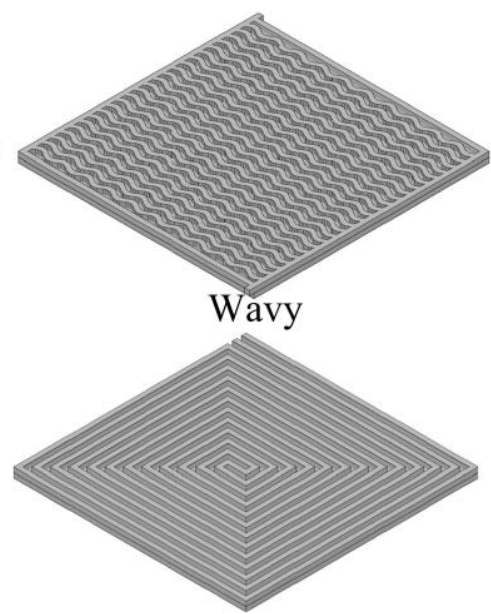

Coil outer inlet

Figure 8. Evaluated cooling channel designs [28, 29]. 
The results indicate that for electronic cooling applications, the coiled design provides a higher heat transfer rate and more uniform temperature distribution as inferred in Figure 9. However, the pressure drop imposed by these channel designs is significantly higher compared to those imposed by rectilinear designs (parallel, wavy, oblique fin). The only rectilinear design with a comparable pressure drop with the coiled design is the serpentine design. However, the serpentine design does not offer a high heat transfer rate and uniform temperature distribution as coiled designs do. To investigate the effect of cooling fluid velocity, five different inlet mass flow rates which correspond to Reynolds numbers of 100, 250, 500, 750 and 1000 were evaluated. The result indicates that cooling performance becomes better as the mass flow rate increases. Surprisingly, design that was found to be superior for mass flow rate can be inferior when a higher mass flow rate is imposed. It was found that the coiled design is more suitable for high mass flow rate applications than for low flow rate applications. In terms of temperature uniformity, it was found that a higher mass flow rate yields a more uniform temperature distribution in the cooling channel.

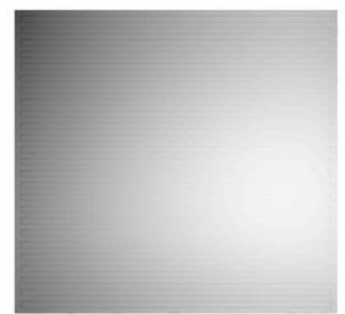

Parallel

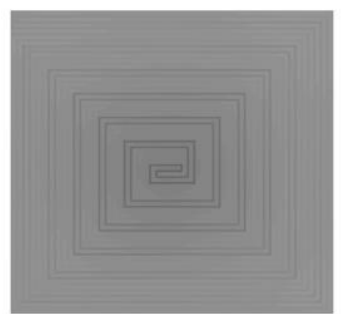

Coil inner inlet

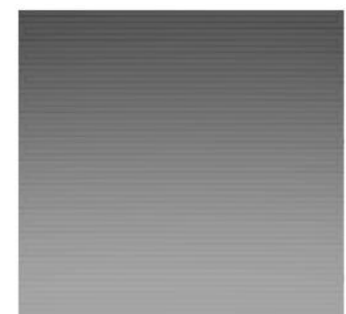

Serpentine

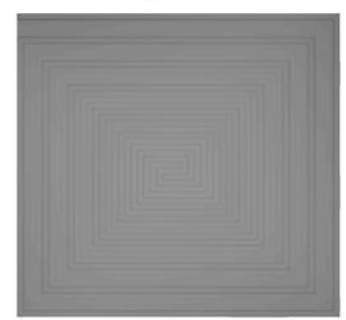

Coil outer inlet

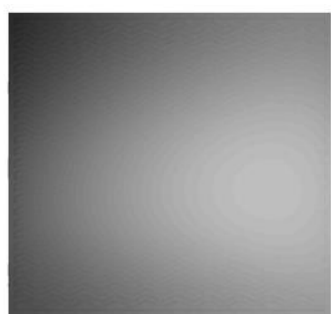

Wavy

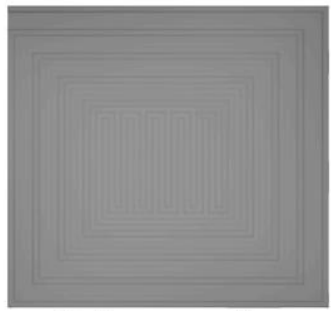

Coil serpentine

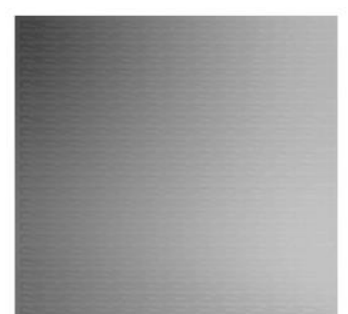

Parallel oblique

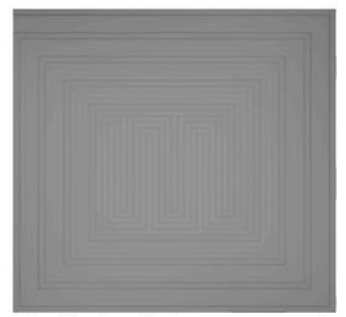

Coil double serpentine

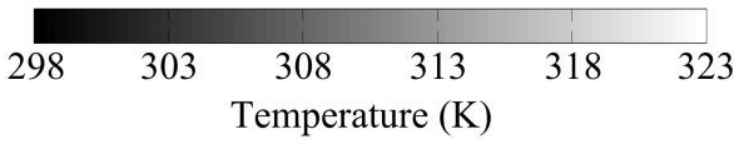

Figure 9. Temperature distribution of the evaluated cooling channel designs [28].

In PEFC cooling applications, interestingly, the coiled design was found to not perform as expected: a hot spot was generated when this design was applied, high liquid water (flooding) was observed, and oxygen depletion was seen in the middle area of the fuel cell. This highlights that the coiled design may not be suitable for PEFC applications. In addition to applications in the heat transfer field, the application of noncircular coiler tubes in the chemical field as micro-reactors has been evaluated and reported [30, 31]. Similar to those of heat transfer, coiled tube reactors offer higher reaction and mixing rates compared to straight tube reactors. The limitation of this study is that a similar liquid channel design is applied for all (cathode, anode and cooling channel) in the co-flow arrangement. Future studies should evaluate combinations of various liquid channels and various arrangements, i.e. counter flow and cross flow. 


\section{Challenges and Research Directions}

In the previous section, the superior heat transfer rate received when using the coiled tube compared to the straight tube was presented and discussed. Those studies highlighted the potential application of non-circular tubes in the thermal engineering field. However, several challenges should be overcome before non-circular coiled tubes can be widely adopted in real heat transfer applications. Some of these challenges are summarized as follows:

- As discussed earlier, higher heat transfer rate offered by non-circular coiled tubes is always accompanied by a higher pressure drop and in turn, requiring a higher pumping power. Studies on coil curvature reveal that smaller curvatures may increase heat transfer, but significantly increase pressure drop such that it has the lowest FoM. Therefore, future studies should be conducted to investigate the optimum curvature radius which offers a high heat transfer rate while keeping the pumping power at the minimum.

- While the presented studies have indicated that the non-circular tube offers high heat transfer rate, manufacturing these tubes may be not economically feasible due to the complexity involved and high manufacturing costs incurred. A life cycle analysis is needed to examine the economic feasibility of non-circular tubes in real applications. An advanced manufacturing technique may be able to reduce the manufacturing cost to an economic level.

- In the cross-section investigation, it was found that non-circular tubes yield higher heat transfer rates as compared to circular tubes. It should be noted, however, that non-circular cross-section may impose a structural issue. These cross-section geometries may not be able to withstand the operating pressure of a real heat exchanger. Hence, further studies evaluating the strength and integrity of these tubes are necessary.

- For applications encountering gas-solid particle flow, non-circular coiled tubes may suffer high erosion and corrosion especially at the outer wall where velocity is higher and secondary flow is observed. For this application, a coating on the outer wall may be considered. Studies investigating this issue will provide guidelines on the application of non-circular coiled tubes in heat exchanger with gas-solid stream.

- As coiled geometry proposed become more complex, an advanced mesh generation technique is needed. A good mesh is necessary to ensure that the computational results are valid and sounds. However, high numbers of mesh element may incur high computational costs. Hence, keeping the optimum number of mesh is necessary for this study. This can be an interesting topic for a computational engineering study.

As coiled tubes have gained significant attention from researchers worldwide, especially in the recent two to three years, it is expected that more studies on coiled tubes and its heat transfer performance will be conducted and reported. Here are some topics that are expected to be studied in coiled tube heat transfer:

- Extended surface and corrugation. These traditional improvement methods have been and are expected to be the most adopted methods in future studies. Recently, several novel and unique extended surface and corrugated surface designs have been evaluated. It is expected that more new designs will be reported in the near future. 
- Application of nanofluid and nano-encapsulated-phase change materials (nepcm). Several studies reporting heat transfer enhancement by utilizing a combination of coiled fluid and nanofluid (or nepcm) were reported recently. With numerous nanofluids and nepcm available, it is expected that more studies will be conducted to evaluate the heat transfer performance of these nano suspensions when applied in a coiled tube.

- Application of coiled tubes in micro devices. Due to its ability to offer a better mixing and transport process, coiled tubes have the potential to be used in micro device applications. Hence, it is expected that more research on these topic will be conducted and reported.

In addition, investigation on the performance of non-circular coiled tubes for specific applications (heat exchanger, mixer and reactor) will further enhance knowledge in this field and provide general guidelines on the utilization of coiled tubes in the stated applications.

\section{CONCLUSIONS}

For decades, coiled tubes have been one of major focus in heat transfer engineering due to its high heat transfer rates. Significant efforts have been devoted to investigate and improve the heat transfer performance of coiled tubes. While most studies on coiled tubes are concentrated on circular tubes, few studies had dealt with non-circular tubes. In the last couple years, a string of computational studies have been conducted to investigate the heat transfer performance of non-circular coiled tubes and various key parameters affecting it such as coil geometry, cross-section, coil curvature, Reynolds number, and boundary conditions. This paper is intended to extract important information from these studies, highlight important findings and provide guidelines for future studies and applications of non-circular coiled tubes in thermal engineering. In summary, a high pumping power requirement is found to be the major drawback of noncircular coiled tubes. Considerable efforts are required to carefully address and overcome this issue. Other issues that need to be addressed are manufacturing complexity, structural issue and mesh generation for complex geometries. Moreover, for applications involving gas-solid particle flow, the used non-circular coiled tube may suffer high erosion and corrosion especially at the outer wall where velocity is higher and secondary flow is observed. As such more studies on this topic are necessary. On the application side, more studies may be directed towards the application of coiled tubes in micro or nano scale for micro device applications.

\section{ACKNOWLEDGEMENTS}

The authors would like to acknowledge Universiti Teknologi PETRONAS, McGill University and National University of Singapore for the facilities and support in conducting this research.

\section{REFERENCES}

[1] Abdolbaqi MK, Azwadi C, Mamat R, Azmi W, Najafi G. Nanofluids heat transfer enhancement through straight channel under turbulent flow. International Journal of Automotive and Mechanical Engineering. 2015;11:2294-305. 
[2] Huminic G, Huminic A. Heat transfer and entropy generation analyses of nanofluids in helically coiled tube-in-tube heat exchangers. International Communications in Heat and Mass Transfer. 2016;71:118-25.

[3] Xing Y, Zhong F, Zhang X. Numerical study of turbulent flow and convective heat transfer characteristics in helical rectangular ducts. Journal of Heat Transfer. 2014;136:121701, 1-6.

[4] Dean W. XVI. Note on the motion of fluid in a curved pipe. The London, Edinburgh, and Dublin Philosophical Magazine and Journal of Science. 1927;4:208-23.

[5] Dean W. LXXII. The stream-line motion of fluid in a curved pipe (Second paper). The London, Edinburgh, and Dublin Philosophical Magazine and Journal of Science. 1928;5:673-95.

[6] Masliyah JH. On laminar flow in curved semicircular ducts. Journal of Fluid Mechanics. 1980;99:469-79.

[7] Dennis S. Dual solutions for steady laminar flow through a curved tube. Quart J Mech Appl Math. 1982;35:305-24.

[8] Dravid AN, Smith K, Merrill E, Brian P. Effect of secondary fluid motion on laminar flow heat transfer in helically coiled tubes. AIChE Journal. 1971;17:1114-22.

[9] Akiyama M, Cheng K. Graetz problem in curved pipes with uniform wall heat flux. Applied Scientific Research. 1974;29:401-18.

[10] Kumar V, Gupta P, Nigam K. Fluid flow and heat transfer in curved tubes with temperature-dependent properties. Industrial \& engineering chemistry research. 2007;46:3226-36.

[11] Shokouhmand H, Salimpour M. Optimal Reynolds number of laminar forced convection in a helical tube subjected to uniform wall temperature. International Communications in Heat and Mass Transfer. 2007;34:753-61.

[12] Liou T. Flow visualization and LDV measurement of fully developed laminar flow in helically coiled tubes. Experiments in fluids. 1992;13:332-8.

[13] Naphon P, Suwagrai J. Effect of curvature ratios on the heat transfer and flow developments in the horizontal spirally coiled tubes. International Journal of Heat and Mass Transfer. 2007;50:444-51.

[14] Norouzi M, Kayhani M, Nobari M, Demneh MK. Convective heat transfer of viscoelastic flow in a curved duct. World Acad Sci Eng Technol. 2009;56:32733.

[15] Rennie TJ, Raghavan VG. Experimental studies of a double-pipe helical heat exchanger. Experimental Thermal and Fluid Science. 2005;29:919-24.

[16] Rennie TJ, Raghavan VG. Numerical studies of a double-pipe helical heat exchanger. Applied Thermal Engineering. 2006;26:1266-73.

[17] Zachár A. Analysis of coiled-tube heat exchangers to improve heat transfer rate with spirally corrugated wall. International Journal of Heat and Mass Transfer. 2010;53:3928-39.

[18] Egner MW, Burmeister LC. Heat transfer for laminar flow in spiral ducts of rectangular cross section. Transactions of the ASME-C-Journal of Heat Transfer. 2005;127:352, 1-5.

[19] Ko T-H. A numerical study on entropy generation and optimization for laminar forced convection in a rectangular curved duct with longitudinal ribs. International Journal of Thermal Sciences. 2006;45:1113-25. 
[20] Kurnia JC, Sasmito AP, Mujumdar AS. Evaluation of the heat transfer performance of helical coils of non-circular tubes. Journal of Zhejiang University-SCIENCE A. 2011;12:63-70.

[21] Sasmito AP, Kurnia JC, Wang W, Jangam SV, Mujumdar AS. Numerical analysis of laminar heat transfer performance of in-plane spiral ducts with various cross-sections at fixed cross-section area. International Journal of Heat and Mass Transfer. 2012;55:5882-90.

[22] Kurnia JC, Sasmito AP, Mujumdar AS. Laminar convective heat transfer for inplane spiral coils of noncircular cross sections ducts: A computational fluid dynamics study. Thermal science. 2012;16:109-18.

[23] Sasmito AP, Kurnia JC, Mujumdar AS. Numerical evaluation of laminar heat transfer enhancement in nanofluid flow in coiled square tubes. Nanoscale Research Letters. 2011;6:376.

[24] Kurnia JC, Sasmito AP, Jangam SV, Mujumdar AS. Heat transfer in coiled square tubes for laminar flow of slurry of microencapsulated phase change material. Heat Transfer Engineering. 2013;34:994-1007.

[25] Kurnia JC, Sasmito AP, Mujumdar AS. Laminar heat transfer performance of power law fluids in coiled square tube with various configurations. International Communications in Heat and Mass Transfer. 2014;57:100-8.

[26] Kurnia JC, Sasmito AP, Mujumdar AS. Thermal Performance of Coiled Square Tubes at Large Temperature Differences for Heat Exchanger Application. Heat Transfer Engineering. 2016;37:1341-56.

[27] Kurnia JC, Sasmito AP, Shamim T, Mujumdar AS. Numerical investigation of heat transfer and entropy generation of laminar flow in helical tubes with various cross sections. Applied Thermal Engineering. 2016;102:849-60.

[28] Kurnia JC, Sasmito AP, Mujumdar AS. Numerical investigation of laminar heat transfer performance of various cooling channel designs. Applied Thermal Engineering. 2011;31:1293-304.

[29] Sasmito AP, Kurnia JC, Mujumdar AS. Numerical evaluation of various gas and coolant channel designs for high performance liquid-cooled proton exchange membrane fuel cell stacks. Energy. 2012;44:278-91.

[30] Sasmito AP, Kurnia JC, Mujumdar AS. Numerical evaluation of transport phenomena in a $\mathrm{T}$-junction microreactor with coils of different configurations. Industrial \& Engineering Chemistry Research. 2011;51:1970-80.

[31] Kurnia JC, Sasmito AP, Birgersson E, Shamim T, Mujumdar AS. Evaluation of mass transport performance in heterogeneous gaseous in-plane spiral reactors with various cross-section geometries at fixed cross-section area. Chemical Engineering and Processing: Process Intensification. 2014;82:101-11.

[32] Ramasamy D, Kadirgama K, Rahman MM, Zainal ZA. Analysis of compressed natural gas burn rate and flame propagation on a sub-compact vehicle engine. International Journal of Automotive and Mechanical Engineering. 2015;11:2405-16.

[33] Edwards DK, Denny VE, Mills AF. Transfer processes. an introduction to diffusion, convection and radiation. Series in Thermal and Fluids Engineering, New York: McGraw-Hill, and Washington: Hemisphere Publication Co, 1978, 2nd ed. 1978;1.

[34] Baehr D, Stephan K. Heat and Mass Transfer (revised second ed.) SpringerVerlag. Berlin; 2006. 
[35] Manlapaz RL, Churchill SW. Fully developed laminar flow in a helically coiled tube of finite pitch. Chemical Engineering Communications. 1980;7:57-78.

[36] Ramasamy D, Yuan GC, Bakar RA, Zainal ZA. Validation of road load characteristic of a sub-compact vehicle by engine operation. International Journal of Automotive and Mechanical Engineering. 2014;9:1820-31.

[37] Ramasamy D, Zainal Z, Kadirgama K, Briggs HW-G. Effect of dissimilar valve lift on a bi-fuel CNG engine operation. Energy. 2016;112:509-19.

[38] Kays WM, Crawford ME, Weigand B. Convective heat and mass transfer: Tata McGraw-Hill Education; 2012.

[39] Fluent A. 14.5: User's guide, ANSYS. Inc, Canonsburg, PA. 2013.

[40] L Syam S, KV S. An experimental study on heat transfer and friction factor of Al2O3 nanofluid. Journal of Mechanical Engineering and Sciences 2011;1:99112.

[41] Mahendran M, Lee GC, Sharma K, Shahrani A. Performance evaluation of evacuated tube solar collector using water-based titanium oxide (Tio2) nanofluid. Journal of Mechanical Engineering and Sciences 2012;3:301-10.

[42] Adnan Mohammed H, KV S, RA B. Heat transfer enhancement with nanofluids-a review. Journal of Mechanical Engineering and Sciences 2013;4:452-61.

[43] Vajjha RS, Das DK, Namburu PK. Numerical study of fluid dynamic and heat transfer performance of $\mathrm{Al} 2 \mathrm{O} 3$ and $\mathrm{CuO}$ nanofluids in the flat tubes of a radiator. International Journal of Heat and fluid flow. 2010;31:613-21.

[44] Hussein AM, Bakar RA, Kadirgama K, Sharma K. Experimental measurement of nanofluids thermal properties. International Journal of Automotive and Mechanical Engineering. 2013;7:850-63.

[45] Sharma KV, L Syam S. Laminar convective heat transfer and friction factor of A12O3 nanofluid in circular tube fitted with twisted tape inserts. International Journal of Automotive and Mechanical Engineering 2011;3:265-78.

[46] Srinivasa RG, Sharma KV, P CS, Bakar RA, Rahman MM, Noor MM. Experimental study on heat transfer coefficient and friction factor of $\mathrm{Al} 2 \mathrm{O} 3$ nanofluid in a packed bed column. Journal of Mechanical Engineering and Sciences. 2011;1:1-15.

[47] Ravisankar B, Chand VT. Influence of nanoparticle volume fraction, particle size and temperature on thermal conductivity and viscosity of nanofluids-A review. International Journal of Automotive and Mechanical Engineering. 2013;8:1316-38. 